\title{
BROWNIAN LOCAL TIMES AND TABOO PROCESSES
}

\author{
BY \\ FRANK B. KNIGHT
}

0. Introduction. This paper is an application of the Markov property of the Brownian local times, as obtained in ([4], [6]; see also [10]), to the study of the sojourn time distributions of the one-dimensional Brownian motion (Wiener process). We recall the following two facts. If $X(t), X(0)=0$, denotes the Wiener process (with continuous path functions),

$$
f(t, x, w)=\frac{d}{d x} \int_{0}^{t} I_{(-\infty, x)}(X(s)) d s
$$

is the "local time" (which by [9] exists and is continuous in $(t, x)$ except for an exceptional set of paths having probability 0$)$, and if for $\alpha>0$ we set $T_{a}(\alpha)=$ $\inf \{t: f(t, a, w)>\alpha\}$ then $f\left(T_{0}(\alpha), x, w\right), x \geqq 0$, is the diffusion process with $x$ as "time parameter," infinitesimal generator of the form $4 y\left(d^{2} / d y\right)^{2}$, initial value $\alpha$, and absorbing barrier at 0 [4, p. 1]. Furthermore, for $a>0, f\left(T_{a}(\alpha), a-x, w\right)$, $0 \leqq x \leqq a$, is the restriction to parameter values in $[0, a]$ of the diffusion process with generator $4 y\left(d^{2} / d y^{2}\right)+2(d / d y)$ and initial value $\alpha$, where 0 is an entrance boundary $[6$, p. 627]; $[4$, p. 62].

It is clear that $T_{a}(0)$ is just the first passage time of $X(t)$ to $a$, and thus the process $f\left(T_{a}(0), a-x, w\right)$, which starts at the entrance boundary 0 , provides information on the sojourn times of $X(t)$ up to the first passage time. This method can be extended to the first passage time to $\{ \pm a\}$, and our main concern will be with the local and sojourn times up to this passage time, denoted by $A_{a}$.

We shall now summarize the results. Set $A_{a}=\inf \{t:|X(t)|=a\}$. Then, for $0 \leqq x \leqq a, f\left(A_{a}, a-x, w\right)+f\left(A_{a},-a+x, w\right)$ is the diffusion with generator $4 y\left(d^{2} / d y^{2}\right)$ $+2(d / d y)$, initial value 0 , and absolute probability densities $(2 x)^{-1} \exp (-y / 2 x)$, $0 \leqq y$. Suppose, now, that $f\left(A_{a}, 0, w\right)=\alpha$ is fixed and given (this is actually a random variable with density $a^{-1} \exp (-y / a)$, as we see by considering the case $x=a$ above). The conditional process $X(s), 0 \leqq s \leqq A_{a}$ then breaks into two independent parts: an initial part up to time $T_{0}(\alpha)$, and a final part during which there is no return to 0 and ending at $\pm a$ (each) with probability $\frac{1}{2}$. The two processes $f\left(T_{0}(\alpha), x, w\right)$ and $f\left(T_{0}(\alpha),-x, w\right), 0 \leqq x \leqq a$, are (conditionally) independent diffusion processes with initial value $\alpha$ which are nonhomogeneous in $x$. Indeed, they are obtained by conditioning $f\left(T_{0}(\alpha), \pm x, w\right)$, when $f\left(A_{a}, 0, w\right)=\alpha$ is not given, by the condition that (respectively) $f\left(T_{0}(\alpha), \pm a, w\right)=0$. Their transition function,

Received by the editors October 9, 1968. 
like that of the unconditional (homogeneous) process, can be given explicitly. The total sojourn time $\int_{0}^{a} f\left(T_{0}(\alpha), x, w\right) d x$, under the same conditioning, has the density with Laplace transform $\exp (\alpha / 2 a)\left(1-a(2 \lambda)^{1 / 2} \operatorname{cotanh} a(2 \lambda)^{1 / 2}\right)$. We are not able to invert this transform explicitly, but after squaring and integrating out on $\alpha$ we obtain the transform of the last return time to 0 before $A_{a}$. It is

$$
\left(a(2 \lambda)^{1 / 2}\right)^{-1} \tanh a(2 \lambda)^{1 / 2},
$$

and can be inverted in terms of $\theta$-functions.

As for the part of the process between this last return time $T_{0}(\alpha)$ (conditionally, as before) and $A_{a}$, its properties are obviously free of the given value of $\alpha$. Denoting the corresponding local time process by

$$
f\left(A_{a}, a-x, w\right)-f\left(T_{0}(\alpha), a-x, w\right), \quad 0 \leqq x \leqq a,
$$

where $\alpha$ is arbitrary but fixed, if one is also given that $X\left(A_{a}\right)=a$ (given $-a$ instead the process would vanish identically) this process becomes the nonhomogeneous diffusion obtained by conditioning the one with generator $4 y\left(d^{2} / d y^{2}\right)+2(d / d y)$ and initial value 0 by the requirement that it also vanish when $x=a$. Its distributions are obtained explicitly. Finally, combining the two parts of the process, we obtain the explicit distributions of $f\left(A_{a}, x, w\right)$ without any conditioning. These have a simple form (Theorem 1.4).

To return to the sojourn times in $(0, a)$, that of the second part of our conditional process is found to have the density with transform $a(2 \lambda)^{1 / 2} \operatorname{cosech} a(2 \lambda)^{1 / 2}$, which again can be inverted using $\theta$-functions. We note that the product of this transform and of the transform for the last return time to 0 before $A_{a}$ is just $\operatorname{sech} a(2 \lambda)^{1 / 2}$, which gives the (known) distribution of $A_{a}$. Consider next the diffusion $f\left(T_{0}(\alpha), x, w\right)$ with generator $4 y\left(d^{2} / d y^{2}\right)$. If $f\left(T_{0}(\alpha), a, w\right)=\beta$ is fixed and given, then the sojourn $\int_{0}^{a} f\left(T_{0}(\alpha), x, w\right) d x$ has a density whose transform is obtained, but is excessively complicated. It does lead, however, to the relatively simple expression $\exp -\left(\alpha(\lambda / 2)^{1 / 2} \tanh a(2 \lambda)^{1 / 2}\right)$ for the transform of this random variable when the value of $\beta$ is not given.

In the remainder of the paper an attempt is made to define Brownian motion conditionally upon never reaching $a$, or alternatively $\{ \pm a\}$. A natural idea is to consider $X(t)$ in $(0, T)$ for $T$ fixed, conditionally upon $\max _{0 \leqq t \leqq T} X(t)<a$, or upon $\max _{0 \leqq t \leqq T}|X(t)|<a$, and then to let $T \rightarrow \infty$ to obtain limiting joint distributions. In this way we obtain what we term the "Brownian taboo process" with taboo state $a$, or set $\{ \pm a\}$ (see [1, p. 43] for the origin of our use of the work "taboo"). These processes are respectively the unique diffusion on $(-\infty, a)$ with generator

$$
\frac{1}{2} \frac{d^{2}}{d y^{2}}-\frac{1}{a-y} \frac{d}{d y},
$$

and the unique diffusion on $(-a, a)$ with generator

$$
\frac{1}{2} \frac{d^{2}}{d y^{2}}-\frac{\pi}{2 a} \tan \frac{\pi y}{2 a} \frac{d}{d y}
$$


It seems to be of note that the former is essentially the radial component of Brownian motion in 3-dimension (it becomes this exactly if $X(0)=x<0$ is assumed and the conditioning is on $\left.\min _{0 \leqq t \leqq T} X(t)>0\right)$. However, some care is necessary in interpreting these results, since the given conditions take on vanishing probability as $T \rightarrow \infty$. We conclude by observing that if we replace the condition

$$
\max _{0 \leqq t \leqq T}|X(t)|<a \quad \text { by } \max _{0 \leqq t \leqq T_{0}(\alpha)}|X(t)|<a,
$$

and let $\alpha \rightarrow \infty$, we do not recover the taboo process with set $\{ \pm a\}$ but obtain the process with generator

$$
\frac{1}{2} \frac{d^{2}}{d y^{2}}-\frac{\operatorname{sn}(y)}{a-|y|} \frac{d}{d y}
$$

which is similar to the taboo process with taboo state $a$ in $(0, a)$, and to its negative in $(-a, 0)$.

1. The local time processes. The first result given in the Introduction is an easy consequence of the facts stated there.

THEOREM 1.1. The process $f\left(A_{a}, a-x, w\right)+f\left(A_{a},-a+x, w\right), 0 \leqq x \leqq a$, is the diffusion with generator of the form $4 y\left(d^{2} / d y^{2}\right)+2(d / d y)$ and initial state 0 (which is an entrance boundary). Its distributions ( $x$ fixed) have the densities

$$
(2 x)^{-1} \exp (-y / 2 x), \quad 0 \leqq y .
$$

Its transition density is given by

$$
p_{1}\left(x ; y_{1} y_{2}\right)=\frac{y_{1} y_{2}}{8 x^{3}} \exp \left(-\frac{y_{1} y_{2}}{2 x}\right) \sum_{k=0}^{\infty}((k+1) !)^{-2}\left(\frac{y_{1} y_{2}}{2 x^{2}}\right)^{k}+\frac{\exp \left(-y_{1} y_{2} / 2 x\right)}{2 x} .
$$

Proof. It is obvious that the process defines the local time of $|X(T)|$ at $a-x$ up to the first passage time of $|X(t)|$ to $a$ (provided that the local time at 0 is defined as the right derivative only). By a known result $[3, \mathrm{pp} .81,82]$ the process $|X(t)|$ can be constructed (up to stochastic equivalence) by deleting from $X(t)$ the negative excursions and then collapsing the $t$-axis to the left to fill up the resulting gaps. It is therefore clear that the local time of $|X(t)|$ at points $0 \leqq a-x$ is stochastically the same as that of $X(t)$ up to its first passage time to $a$. This reduces the theorem to known properties of the latter $([4$, p. 75 , line 1$]$ where the formula given is for $\frac{1}{2}$ of the present process). Indeed the absolute probabilities follow from the transition probabilities by setting $y_{1}=0$.

We suppose now that $f\left(A_{a}, 0, w\right)=\alpha$ is given. Thus in particular $T_{0}(\alpha)<A_{a}$ and we can consider the conditional processes $f\left(T_{0}(\alpha), x, w\right)$ and $f\left(T_{0}(\alpha),-x, w\right)$, $0 \leqq x \leqq a$, which are clearly equivalent in the stochastic sense. Let the path segments $X(s), 0 \leqq s \leqq A_{a}$, be divided into the two parts $0 \leqq s \leqq T_{0}(\alpha)$ and $T_{0}(\alpha) \leqq s \leqq A_{a}$. It is clear by considering the reversed process $X\left(A_{a}-s\right), 0 \leqq s \leqq A_{a}-T_{0}(\alpha)[5$, p. 212] that the segment $X\left(T_{0}(\alpha)+s\right), 0 \leqq s \leqq A_{a}-T_{0}(\alpha)$, is (conditionally) independent of 
the past, and (stochastically) free of the given value $\alpha$. The segment $X(s), 0 \leqq s \leqq A_{a}$, can be reconstructed by conditioning $X(t)$ in $\left[0, T_{0}(\alpha)\right]$ by $\max _{0 \leqq s \leqq T_{0}(\alpha)}|X(s)|<a$, without assuming $f\left(A_{a}, 0, w\right)=\alpha$, and then adjoining an independent section with the distributions of $X\left(T_{0}(\alpha)+s\right), 0 \leqq s \leqq A_{a}-T_{0}(\alpha)$, conditionally upon $f\left(A_{a}, 0, w\right)$ $=\alpha$. But it is clear that in $\left[0, T_{0}(\alpha)\right]$ the condition $\max _{0 \leqq s \leqq T_{0}(\alpha)} X(s)<a$ is equivalent to $f\left(T_{0}(\alpha), a, w\right)=0$, since 0 is an absorbing boundary for $f\left(T_{0}(\alpha), x, w\right)$. We introduce the notation

$$
\begin{aligned}
p_{0}\left(x ; y_{1}, y_{2}\right) & =\frac{y_{1}}{4 x^{2}} \exp \left(-\frac{y_{1}+y_{2}}{2 x}\right) \sum_{k=0}^{\infty} \frac{1}{k !(k+1) !}\left(\frac{y_{1} y_{2}}{4 x^{2}}\right)^{k} \\
& =\frac{\exp \left(-\left(y_{1}+y_{2}\right) / 2 x\right)}{2 i x}\left(\frac{y_{1}}{y_{2}}\right)^{1 / 2} J_{1}\left(\frac{i\left(y_{1} y_{2}\right)^{1 / 2}}{x}\right)
\end{aligned}
$$

for the transition density of $f\left(T_{0}(\alpha), x, w\right)$, which also has an absorption probability $P_{0}\left(x ; y_{1},\{0\}\right)=\exp \left(-y_{1} / 2 x\right)$ [4, Theorem 1.2$]$. Then we have immediately

THEOREM 1.2. The transition density of the conditional local time process $f\left(T_{0}(\alpha), x, w\right), 0 \leqq x \leqq a$, given $f\left(A_{a}, 0, w\right)=\alpha$, is

$$
\begin{aligned}
p\left(x_{1}, y_{1} ; x_{2}, y_{2}\right) & =p_{0}\left(x_{2}-x_{1} ; y_{1}, y_{2}\right) \frac{P_{0}\left(a-x_{2} ; y_{2},\{0\}\right)}{P_{0}\left(a-x_{1} ; y_{1},\{0\}\right)} \\
& =\exp \left(-\frac{y_{1}\left(a-x_{2}\right)^{2}+y_{2}\left(a-x_{1}\right)^{2}}{2\left(a-x_{2}\right)\left(a-x_{1}\right)\left(x_{2}-x_{1}\right)}\right) \frac{1}{2 i\left(x_{2}-x_{1}\right)}\left(\frac{y_{1}}{y_{2}}\right)^{1 / 2} J_{1}\left(\frac{i\left(y_{1} y_{2}\right)^{1 / 2}}{x_{2}-x_{1}}\right),
\end{aligned}
$$

with an absorbtion probability $\exp \left(-y_{1}\left(a-x_{2}\right) / 2\left(a-x_{1}\right)\left(x_{2}-x_{1}\right)\right)$. The marginal probabilities have the density

$$
\exp \left(-\frac{\alpha(a-x)^{2}+y a^{2}}{2 a x(a-x)}\right) \frac{1}{2 i x} J_{1}\left(\frac{i(\alpha y)^{1 / 2}}{x}\right)\left(\frac{\alpha}{y}\right)^{1 / 2}, \quad 0 \leqq y
$$

and a mass at 0 of magnitude $\exp (-\alpha(a-x) / 2 a x)$.

Proof. The only point requiring comment is that the marginal probabilities are obtained from the transition probabilities by setting $x_{1}=0, x_{2}=x, y_{1}=\alpha$, and $y_{2}=y$.

It is clear from the above and the independence of the (unconditional) processes $f\left(T_{0}(\alpha), \pm x, w\right)$, which is evident from [4], that the conditional ones are also independent, and the density $a^{-1} \exp (-y / a)$ for the conditioning random variable $\alpha$ follows from Theorem 1.1 upon setting $x=a$, as noted earlier.

From the independence of $X\left(T_{0}(\alpha)+s\right)$ and the "past," together with the Markov properties of the $f\left(T_{0}(\alpha), x, w\right)$ and $f\left(A_{a}, a-x, w\right)$, it follows that conditional upon $f\left(A_{a}, 0, w\right)=\alpha$ the process $f\left(A_{a}, a-x, w\right)-f\left(T_{0}(\alpha), a-x, w\right)$ is also a diffusion (with distributions free of $\alpha$ ) and it becomes obvious that its structure is as follows.

THEOREM 1.3. The conditional process $f\left(A_{a}, a-x, w\right)-f\left(T_{0}(\alpha), a-x, w\right), 0 \leqq x \leqq a$, is obtained by conditioning the diffusion with generator $4 y\left(d^{2} / d y^{2}\right)+2(d / d y)$ and initial value 0 to take on the value 0 when $x=a$ (since 0 is inaccessible the conditioning is 
understood as the limit, given values $\varepsilon>0$ for $x=a$, as $\varepsilon \rightarrow 0+)$. The marginal distributions of this conditional diffusion have the densities

$$
\frac{a}{2 x(a-x)} \exp -\frac{y a}{2 x(a-x)}, \quad 0 \leqq y .
$$

REMARK. We shall not write the transition function.

Proof. One simply uses the expression for $p_{1}$ (Theorem 1.1) with $y_{1}$ or $y_{2}=0$ to obtain

$$
\left(\frac{1}{2 x} \exp -\frac{y}{2 x}\right)\left(\frac{1}{2(a-x)} \exp -\frac{y}{2(a-x)}\right) 2 a,
$$

which equals the stated density.

Let us consider finally the densities for the (unconditional) process $f\left(A_{a}, x, w\right)$, which is non-Markovian but becomes Markovian if $X\left(A_{a}\right)=a$ or $X\left(A_{a}\right)=-a$ is given $[4$, p. 75$]$. We obtain the

THEOREM 1.4. $f\left(A_{a}, x, w\right),-a<x<a$, has the distribution with density

$$
\frac{a^{2}}{(a+|x|)\left(a^{2}-x^{2}\right)} \exp -\frac{y a}{a^{2}-x^{2}}, \quad 0<y,
$$

together with a probability mass at 0 of magnitude $|x| /(a+|x|)$.

Proof. Let $f_{x}(y)$ denote the density of $f\left(A_{a}, x, w\right)$ conditional upon $X\left(A_{a}\right)=-a$. Then clearly the desired density will be $\frac{1}{2}\left(f_{x}(y)+f_{-x}(y)\right)$ with an additional mass at 0 of size $\frac{1}{2}\left(1-\int_{0}^{\infty} f_{|x|}(y) d y\right)$. Choosing $0<x<a$, we note from Theorem 1.3 that

$$
f_{-x}(y)=f_{x}(y) *\left(\frac{a}{2 x(a-x)} \exp -\frac{y a}{2 x(a-x)}\right),
$$

where the asterisk denotes convolution. But $f_{x}$ may be computed using the known density $a^{-1} \exp (-\alpha / a)$ for $f_{0}(\alpha)$, which is unaffected by the conditioning. We must simply condition the transition $p_{0}$ from 0 to $x$ by $f\left(A_{a}, a, w\right)=0$, as in Theorem 1.2. From the series expression for $p_{0}$ we evaluate easily

$$
\begin{aligned}
f_{x}(y) & =\int_{0}^{\infty} \frac{1}{a}\left(\exp -\frac{\alpha}{a}\right) \frac{\alpha}{4 x^{2}} \exp \left(-\frac{\alpha+y}{2 x}-\frac{y}{2(a-x)}+\frac{\alpha}{2 a}\right) \sum_{k=0}^{\infty} \frac{1}{k !(k+1) !}\left(\frac{\alpha y}{4 x^{2}}\right)^{k} d \alpha \\
& =\frac{a}{(a+x)^{2}} \exp -\frac{y a}{a^{2}-x^{2}}, \quad 0<y .
\end{aligned}
$$

There is also a mass at 0 given by

$$
\int_{0}^{\infty} \frac{1}{a} \exp \left(-\frac{\alpha}{a}-\frac{\alpha}{2 x}+\frac{\alpha}{2 a}\right) d \alpha=\frac{2 x}{x+a} .
$$

It is now a routine matter to form the convolution for $f_{-x}$, and we obtain the result that

$$
f_{-x}(y)=\frac{a}{a^{2}-x^{2}} \exp -\frac{y a}{a^{2}-x^{2}}, \quad 0<y .
$$

Forming the average of $f_{ \pm}(y)$ now completes the proof. 
REMARK. We indicated in [4, pp. 74, 75] a different method for obtaining this result, but did not anticipate that the outcome would be simple.

REMARK. The results of this section apply without change to any conservative diffusion process which, in the universal scale, is regular in $(-a, a)$ and is absorbed at $\{ \pm a\}$. Indeed, such a diffusion has a generator of the form $(d / d m)\left(d^{+} / d x^{+}\right)$ where $m$ is an increasing, right-continuous function on $(-a, a)$. By a theorem of Ito and McKean [3, \$5.2] it may be obtained from Brownian motion by a random time change, in such a way that (a) the local times at equal points $y$ and at corresponding times $t$ and $\tau$ are identical if the local time for a diffusion $Y(t)$ is defined by

$$
2 \frac{d}{d m} \int_{0}^{t} I_{(-\infty, y)}(Y(s)) d s
$$

and (b) the first passage times to $\{ \pm a\}$ for the two processes correspond to each other under the random time change $[4, \mathrm{pp} .83,84]$. Thus in order to interpret the results of this section for the general diffusion $Y(t)$ one has only to define

$$
f(t, y, w)=2 \frac{d}{d m(y)} \int_{0}^{t} I_{(\infty, y)}(Y(s)) d s
$$

and the results hold exactly as stated. (Note that this also changes the definition of $\left.T_{0}(\alpha)\right)$.

2. Some sojourn times. We next take up the integrals in $x$ of these local time processes. To obtain their distributions, or the Laplace transforms thereof, it seems easiest to use not the processes directly but rather an approximation in terms of symmetric Bernoulli trials.

THEOREM 2.1. The Laplace transform of the conditional density of

$$
\int_{0}^{a} f\left(T_{0}(\alpha), x, w\right) d x
$$

given that $f\left(A_{a}, 0, w\right)=\alpha$ is given by

$$
\exp \left(\alpha / 2 a-\alpha(\lambda / 2)^{1 / 2} \operatorname{cotanh} a(2 \lambda)^{1 / 2}\right) .
$$

That of $\int_{0}^{a}\left(f\left(A_{a}, x, w\right)-f\left(T_{0}(\alpha), x, w\right)\right) d x$, under the same conditioning, is

$$
a(2 \lambda)^{1 / 2} \operatorname{cosech} a(2 \lambda)^{1 / 2} .
$$

Proof. We consider in the interval $[0, a]$ an ordinary symmetric random walk on the points $k a / n, 0 \leqq k \leqq n$ with reflecting boundaries 0 and $a$. Let $g_{0, n}\left(\right.$ resp. $\left.g_{a, n}(s)\right)$ denote the generating function of the conditional distribution of the number of steps required to reach 0 (resp. $a$ ) if the process starts at $a / n$ and it is given that 0 (resp. $a$ ) is reached before $a$ (resp. 0 ). Then it follows immediately from the known 
generating functions for the classical "gambler's ruin" problem [2, vol. 1, p. 320] that we have

$$
g_{0, n}(s)=\frac{n}{n-1}\left(\frac{\lambda_{1}^{n-1}(s)-\lambda_{2}^{n-1}(s)}{\lambda_{1}^{n}(s)-\lambda_{2}^{n}(s)}\right)
$$

and

$$
g_{a, n}(s)=n\left(\frac{\lambda_{1}(s)-\lambda_{2}(s)}{\lambda_{1}^{n}(s)-\lambda_{2}^{n}(s)}\right)
$$

where $\lambda_{i}(s)=\left(1 \pm\left(1-s^{2}\right)^{1 / 2}\right) / s$ respectively, $i=1$ or 2 .

Since $g_{0, n}^{k}(s) g_{a, n}(s)$ is the generating function of $-(k+1)$ plus the number of steps of the random walk required to return to 0 exactly $k$ times (starting at 0 ) before reaching $a$ given that 0 is visited exactly $k+1$ times before $a$ (and that from 0 it always returns to $a / n$ in one step), it is plausible that as $k_{n} \rightarrow \infty$ at the correct rate and if $s$ is replaced by $e^{-\lambda r_{n}}$ for suitable $r_{n}$ this expression will converge to the transform of the distribution of the sojourn in $(0, a)$ up to $A_{a}$ given that the local time at 0 is $\alpha$ (corresponding to $k_{n}$ ) and that $X\left(A_{a}\right)=a$. Under the same conditions the factors $g_{0, n}^{k}(s)$ and $g_{a, n}(s)$ will then converge to the two transforms in the theorem. This method is easy to justify rigorously by applying [4, Theorem 4.1] or [8, Theorem 2] where $f(t, x, w)$ is obtained as a uniform limit of the corresponding random walk expressions. (In [4] one has $a=1$ and $2^{n}$ in place of $n$, but this is unimportant. It should be noted, however, that the approximating expressions are sufficiently regular in $\alpha$, uniformly in $n$, to obtain from this convergence that of the conditional transforms when $\alpha$ is fixed.) The necessary substitutions also are seen to be $k_{n}=[\alpha n / 2 a]$ and $s=\exp \left(-\lambda a^{2} / n^{2}\right)$ (the latter since $j$ steps require time $j\left(a^{2} / n^{2}\right)$ ). It is now a routine calculation to let $n \rightarrow \infty$ and obtain the theorem. For brevity we present only the second computation, which is free of $k_{n}$. We have

$$
\begin{aligned}
\lim _{n \rightarrow \infty} n \frac{\lambda_{1}(s)-\lambda_{2}(s)}{\lambda_{1}^{n}(s)-\lambda_{2}^{n}(s)} & =\lim _{n \rightarrow \infty} n \exp \left(\frac{\lambda a^{2}}{n^{2}}-\frac{\lambda a^{2}}{n}\right) \frac{\left(1+a(2 \lambda)^{1 / 2} / n\right)-\left(1-a(2 \lambda)^{1 / 2} / n\right)}{\left(1+a(2 \lambda)^{1 / 2} / n\right)^{n}-\left(1-a(2 \lambda)^{1 / 2} / n\right)^{n}} \\
& =\frac{2 a(2 \lambda)^{1 / 2}}{\exp \left(a(2 \lambda)^{1 / 2}\right)-\exp \left(-a(2 \lambda)^{1 / 2}\right)^{\prime}}
\end{aligned}
$$

as required.

As a further check of this theorem, it is noted that the transform for $A_{a}$ ought to be given by multiplying these two and integrating out on $\alpha$ with respect to its density. This leads to

$(2 \lambda)^{1 / 2} \operatorname{cosech} a(2 \lambda)^{1 / 2} \int_{0}^{\infty} \exp -\left(\alpha / 2 a+\alpha(\lambda / 2)^{1 / 2} \operatorname{cotanh} a(2 \lambda)^{1 / 2}\right) d \alpha=\operatorname{sech} a(2 \lambda)^{1 / 2}$,

' which is known (and can be routinely verified) to be correct. At the same time, if we omit the factor $a(2 \lambda)^{1 / 2} \operatorname{cosech} a(2 \lambda)^{1 / 2}$ we obtain $\left(1 / a(2 \lambda)^{1 / 2}\right) \tanh a(2 \lambda)^{1 / 2}$. This can be inverted [7, p. 283, \#27] to yield 
COROLlaRY 2.1. The density in $t$ for the time of the last visit to 0 before reaching $\{ \pm a\}$ is given by

$$
\frac{1}{2 a^{2}} \theta_{2}\left(0, \frac{t}{2 a^{2}}\right)=\frac{1}{a(2 \pi t)^{1 / 2}} \sum_{n=-\infty}^{\infty}(-1)^{n} \exp -\frac{2 n^{2} a^{2}}{t}, \quad 0<t .
$$

One can also invert $a(2 \lambda)^{1 / 2} \operatorname{cosech} a(2 \lambda)^{1 / 2}[7$, p. 28, \#6], but both here and for Corollary 2.2 below we have not found an inversion for transforms involving $\exp \left(-\lambda^{1 / 2} f\left(\lambda^{1 / 2}\right)\right)$ where $f$ is cotanh or tanh.

Before applying Theorem 2.1 we shall present an extension which, although unwieldy, is of interest in view of the Markov property of $f\left(T_{0}(\alpha), x, w\right)$, and because of its proof and corollary.

THEOREM 2.2. The Laplace transform of the density of $\int_{0}^{a} f\left(T_{0}(\alpha), x, w\right) d x$, conditional upon $f\left(T_{0}(\alpha), a, w\right)=\beta>0$, is given by

$$
\left(\frac{2 \lambda \alpha}{\beta}\right)^{1 / 2} \frac{\operatorname{cosech} a(2 \lambda)^{1 / 2} J_{1}\left(i(2 \lambda \alpha \beta)^{1 / 2} \operatorname{cosech} a(2 \lambda)^{1 / 2}\right)}{2 i p_{0}(a ; \alpha, \beta) \exp \left((\alpha+\beta)(\lambda / 2)^{1 / 2} \operatorname{cotanh} a(2 \lambda)^{1 / 2}\right)},
$$

where $p_{0}$ is as in Theorem 1.2.

Proof. Since $X\left(T_{0}(\alpha)\right)=0$, the segments $X(s), 0 \leqq s \leqq T_{0}(\alpha)$, can be broken into pieces according to the unique (random) partition $0=T_{0}<T_{1}<\cdots<T_{2 k}<T_{0}(\alpha)$ $<T_{2 k+1}$ such that

$$
\begin{aligned}
& X\left(T_{j}\right)=0 ; j \text { even, and } X(t) \neq a ; T_{j} \leqq t<T_{j+1}, j \text { even } \\
& =a ; j \text { odd } \quad \neq 0 ; T_{j} \leqq t<T_{j+1}, j \text { odd. }
\end{aligned}
$$

Let us first obtain the distribution of $k$ when $f\left(T_{0}(\alpha), a, w\right)=\beta$ is given. The function $f(t, a, w), 0 \leqq t \leqq T_{0}(\alpha)$, increases only during the periods $\left[T_{j}, T_{j+1}\right)$ for $j$ odd. If there are $k$ of these periods then $f\left(T_{0}(\alpha), a, w\right)$ becomes the sum of $k$ independent random variables with density $(1 / 2 a) \exp (-y / 2 a)$, and hence has the density

$$
\frac{1}{2 a(k-1) !}\left(\frac{y}{2 a}\right)^{k-1} \exp -\frac{y}{2 a}
$$

[2, vol. II, p. 10]. Since $f\left(T_{0}(\alpha), 0, w\right)=\alpha$ and $f(t, 0, w)$ increases only during $\left[T_{j}, T_{j+1}\right)$ for $j$ even, these increases having the same exponential density, the probability that $\alpha$ should lie between the $k$ th and $(k+1)$ th partial sums is $(1 / k !)(\alpha / 2 a)^{k} \exp (-\alpha / 2 a)$, in accordance with a Poisson distribution [2, Vol. II, Chapter 1, (3.5)]. Thus we can write that the probability of $k$ is proportional to the product of these two quantities, the first at $y=\beta$, which if we set $r=k-1$ is

$$
\frac{\alpha}{4 a^{2} r !(r+1) !}\left(\exp -\frac{\alpha+\beta}{2 r}\right)\left(\frac{\alpha \beta}{4 a^{2}}\right)^{r} \text {. }
$$

But this is simply the $r$ th term in the series for $p_{0}$, which explains the $p_{0}$ in the denominator of the theorem as a normalization for summation over the probabilities of $k$ (it also explains the terms in the series for $p_{0}$ ). 
Assuming now that $k$ is also given, we see that the increases in $f(t, a, w)$ subdivide $(0, \beta)$ into $k$ subintervals in accordance with $k-1$ points chosen uniformly at random in $(0, \beta)$ [2, Vol. II, Chapter $3, \S 3]$. Given these points the sojourn in $(0, a)$ during $\bigcup_{0<j \text { odd }}^{2 k-1}\left(T_{j}, T_{j+1}\right)$ has the distribution with transform

$$
\left(a(2 \lambda)^{1 / 2} \operatorname{cosech} a(2 \lambda)^{1 / 2}\right)^{k} \exp (\beta / 2 a)\left(1-a(2 \lambda)^{1 / 2} \tanh a(2 \lambda)^{1 / 2}\right)
$$

in accordance with Theorem 2.1, where the exponentials have the fixed product for any choice of the subdividing points. Similarly, the sojourn during

$$
\bigcup_{0 \leqq j \text { even }}^{2(k-1)}\left(T_{j}, T_{j+1}\right) \cup\left(T_{2 k}, T_{0}(\alpha)\right)
$$

has the transform

$$
\left(a(2 \lambda)^{1 / 2} \operatorname{cosech} a(2 \lambda)^{1 / 2}\right)^{k} \exp (\alpha / 2 a)\left(1-a(2 \lambda)^{1 / 2} \operatorname{cotanh} a(2 \lambda)^{1 / 2}\right) .
$$

Since these two sojourns are conditionally independent given $k$ and have sum $\int_{0}^{a} f\left(T_{0}(\alpha), x, w\right) d x$, we can now write for the unconditional transform

$$
\begin{aligned}
p_{0}^{-1}(a ; \alpha, \beta) \frac{\alpha}{4 a^{2}} \sum_{r=0}^{\infty}\left(\frac{\alpha \beta}{4 a^{2}}\right)^{2 r} \frac{\left(a(2 \lambda)^{1 / 2} \operatorname{cosech} a(2 \lambda)^{1 / 2}\right)^{2 r+2}}{r !(r+1) !} \\
\cdot \exp -(\alpha+\beta)(\lambda / 2)^{1 / 2} \operatorname{cotanh} a(2 \lambda)^{1 / 2}
\end{aligned}
$$

This reduces easily to the statement of the theorem.

It is worthwhile to make an indirect check of this result, and to obtain at the same time the unconditional transform for $\int_{0}^{a} f\left(T_{0}(\alpha), x, w\right) d x$. If $\beta$ is given, this last becomes independent of $\int_{a}^{\infty} f\left(T_{0}(\alpha), x, w\right) d x$, which is easily shown to have the density with transform $\exp -\beta(\lambda / 2)^{1 / 2}$ (this is equivalent to that of $T_{0}(\beta / 2)$, which is equivalent to that of $T_{\beta / 2}(0)$ by a result of P. Lévy [see 3, p. 43], and this last has a known transform [3, p. 26]). Moreover, since $\int_{0}^{\infty} f\left(T_{0}(\alpha), x, w\right) d x$ has the transform $\exp -\alpha(\lambda / 2)^{1 / 2}$ while $p_{0}(a ; \alpha, \beta)$ is the density of $\beta$ with a further mass $p\{\beta=0\}=\exp (-\alpha / 2 a)$, we can multiply the two conditional transforms and integrate out on $\beta$ to get $\exp -\alpha(\lambda / 2)^{1 / 2}$. The integration from $0+$ to $\infty$ is easily performed (one can use $\int_{0+}^{\infty} p_{0}(a ; \alpha, \beta) d \beta=1-\exp (-\alpha / 2 a)$ here) and yields

$$
\begin{aligned}
\left(\exp -\alpha\left(\frac{\lambda}{2}\right)^{1 / 2} \operatorname{cotanh} a(2 \lambda)^{1 / 2}\right) & \left(\exp \alpha\left(\frac{\lambda}{2}\right)^{1 / 2} \frac{\operatorname{cosech}^{2} a(2 \lambda)^{1 / 2}}{\operatorname{cotanh} a(2 \lambda)^{1 / 2}+1}-1\right) \\
& =\exp \left(-\alpha\left(\frac{\lambda}{2}\right)^{1 / 2}\right)-\exp \left(-\alpha\left(\frac{\lambda}{2}\right)^{1 / 2} \operatorname{cotanh} a(2 \lambda)^{1 / 2}\right) .
\end{aligned}
$$

But by Theorem 2.1 the added term for $\beta=0$ is exactly the negative of the 2 nd term here, as required. If, on the other hand, we omit the factor $\exp \left(-\beta(\lambda / 2)^{1 / 2}\right)$, we can obtain 
COROLlaRY 2.2. The transform of the density of $\int_{0}^{a} f\left(T_{0}(\alpha), x, w\right) d x$ is given by $\exp \left(-\alpha(\lambda / 2)^{1 / 2} \tanh a(2 \lambda)^{1 / 2}\right)$.

Proof. One obtains directly the expression

$$
\left(\exp -\alpha\left(\frac{\lambda}{2}\right)^{1 / 2} \operatorname{cotanh} a(2 \lambda)^{1 / 2}\right)\left(\exp \alpha\left(\frac{\lambda}{2}\right)^{1 / 2} \frac{\operatorname{cosech}^{2} a(2 \lambda)^{1 / 2}}{\operatorname{cotanh} a(2 \lambda)^{1 / 2}}\right)
$$

which reduces easily to the assertion.

Needless to point out, one can similarly obtain the joint transform of

$$
\int_{0}^{a} f\left(T_{0}(\alpha), x, w\right) d x \text { and } \int_{a}^{\infty} f\left(T_{0}(\alpha), x, w\right) d x
$$

as well as its multidimensional analog, but they seem excessively complicated.

3. Brownian taboo processes. We have studied the local time of $X(t)$ up to the passage times $T_{a}(0)$ and $A_{a}$, thus obtaining some features of the process up until it reaches $a$ or $\{ \pm a\}$. This leaves open the possibility that $a$ or $\{ \pm a\}$ are not reached. One knows, of course, that the corresponding sets have 0 probability, but it is still feasible to define by a limiting procedure the process conditional upon never reaching $a$ or $\{ \pm a\}$. Subject to rigorous justification given below the corresponding definition is

Definition 3.1. The Brownian taboo process with taboo state $a$ (resp. set $\{ \pm a\}$ ) is the diffusion on $(-\infty, a)$ (resp. on $(-a, a)$ ) with joint distributions at times $0 \leqq t_{1}<t_{2}<\cdots<t_{n}$ determined as the weak limits as $T \rightarrow \infty$ of those of $X(t)$ conditional upon $\max _{0 \leqq t \leqq T} X(t)<a$ (resp. upon $\max _{0 \leqq t \leqq T}|X(t)|<a$ ).

REMARK. See [1, p. 43] for a related use of the nomenclature.

We next obtain the existence and structure of these processes.

THEOREM 3.1. The taboo process with taboo state $a$ is the unique diffusion on $(-\infty, a)$ with generator of the form

$$
\frac{1}{2} \frac{d^{2}}{d y^{2}}-\frac{1}{a-y} \frac{d}{d y}
$$

The taboo process with taboo set $\{ \pm a\}$ is the unique diffusion on $(-a, a)$ with generator of the form

$$
\frac{1}{2} \frac{d^{2}}{d y^{2}}-\frac{\pi}{2 a} \tan \frac{\pi y}{2 a} \frac{d}{d y}
$$

REMARK. All of the boundaries for these processes are inaccessible (no boundary conditions are possible). The first process is transient, however, with limit $-\infty$ a.s. as $t \rightarrow \infty$.

Proof. Choose $0=t_{0}<t_{1}<\cdots<t_{n}<T$, and let $p_{a}(t ; x, y)$ and $p_{a,-a}(t ; x, y)$ denote respectively the transition densities of $X(t)$ with absorbing barriers at $a$ or 
at $\pm a$. Let $p_{C, T}$ (resp. $p_{D, T}$ ) denote the joint densities of $X(t)$ given $\max _{0 \leqq t \leqq T} X(t)$ $<a$ (resp. $\max _{0 \leqq t \leqq T}|X(t)|<a$ ). Then setting $x_{0}=0$ it is not hard to see that

$$
\begin{aligned}
p_{C, T}\left(t_{1}, \ldots, t_{n} ; x_{1}, \ldots, x_{n}\right) & \\
& =\prod_{j=1}^{n} p_{a}\left(t_{j}-t_{j-1}, x_{j-1}, x_{j}\right) \frac{P^{x_{n}}\left\{\max _{0<t<T-t_{n}} X(t)<a\right\}}{P^{0}\left\{\max _{0<t<T} X(t)<a\right\}}, \\
p_{D, T}\left(t_{1}, \ldots, t_{n} ; x_{1}, \ldots, x_{n}\right) & \\
& =\prod_{j=1}^{n} p_{a,-a}\left(t_{j}-t_{j-1}, x_{j-1}, x_{j}\right) \frac{P^{x_{n}}\left\{\max _{0 \leqq t \leqq T}|X(t)|<a\right\}}{P^{0}\left\{\max _{0 \leqq t \leqq T}|X(t)|<a\right\}}
\end{aligned}
$$

We also have for $x<a$, [5, Chapter III, §20],

$$
P^{x}\left\{\max _{0 \leqq t \leqq T} X(t)<a\right\}=\left(\frac{2}{\pi}\right)^{1 / 2} \int_{0}^{(a-x) T^{-1 / 2}} \exp -\frac{z^{2}}{2} d z
$$

and for $|x|<a$

$$
\begin{aligned}
P^{x}\left\{\max _{0 \leqq t \leqq T}|X(t)|<a\right\} \\
=\frac{4 a}{\pi} \sum_{k=0}^{\infty}(2 k+1)^{-1} \sin \left(\frac{(2 k+1) \pi(x+a)}{2 a}\right) \exp -\frac{(2 k+1)^{2} \pi^{2} T}{8 a^{2}} .
\end{aligned}
$$

Consequently as $T \rightarrow \infty$ the last factors in (3.1) are easily evaluated:

$$
\lim _{T \rightarrow \infty} \frac{a-x_{n}}{\left(T-t_{n}\right)^{1 / 2}} \frac{\sqrt{ } T}{a}=\frac{a-x_{n}}{a}
$$

and

$$
\lim _{T \rightarrow \infty} \sin \pi \frac{\left(x_{n}+a\right)}{2 a} \exp -\pi^{2} \frac{\left(T-t_{n}\right)}{8 a^{2}} \exp \frac{\pi^{2} T}{8 a^{2}}=\sin \pi \frac{\left(x_{n}+a\right)}{2 a} \exp \frac{\pi^{2} t_{n}}{8 a^{2}}
$$

It follows that as $T \rightarrow \infty$ the joint densities $p_{C, T}$ and $p_{D, T}$ converges respectively to those of the Markov processes with transition densities $p_{a}(t ; x, y)(a-y) /(a-x)$, and

$$
p_{a,-a}(t ; x, y) \frac{\sin (\pi(y+a) / 2 a)}{\sin (\pi(x+a) / 2 a)} \exp \frac{\pi^{2} t}{8 a} .
$$

It remains only to show that these are the transition densities of the stated diffusions. That the paths may be assumed continuous and that the finite boundaries are inaccessible for these processes becomes obvious from their construction. To obtain the form of the generators it then suffices to show that the transition densities satisfy the backward equations

$$
\left(\frac{1}{2} \frac{\partial^{2}}{\partial x^{2}}-\frac{1}{a-x} \frac{\partial}{\partial x}\right) p(t ; x, y)=\frac{\partial}{\partial t} p(t ; x, y)
$$

and

$$
\left(\frac{1}{2} \frac{\partial^{2}}{\partial x^{2}}-\frac{\pi}{2 a} \tan \frac{\pi x}{2 a} \frac{\partial}{\partial x}\right) p(t ; x, y)=\frac{\partial}{\partial t} p(t ; x, y)
$$


respectively. Using the fact that both $p_{a}$ and $p_{a,-a}$ satisfy the equation $\frac{1}{2}\left(\partial^{2} p / \partial x^{2}\right)$ $=\partial p / \partial t$ in their appropriate intervals, the result now follows by straightforward differentiation which may be left to the reader. Finally, the generator

$$
\frac{1}{2} \frac{d^{2}}{d y^{2}}-\frac{1}{a-y} \frac{d}{d y}
$$

corresponds to the Bessel process in 3 dimensions multiplied by -1 and translated by $+a$. It is known [3, p. 133] that this process is transient with a.s. limit $-\infty$, which is inaccessible. This completes the proof $\left({ }^{1}\right)$.

Let us consider, finally, the process $X(t)$ conditioned by $\max _{0 \leqq t \leqq T_{0}(\alpha)}|X(t)|<a$. For $0<\alpha<\beta$ it follows readily from the strong Markov property with stopping time $T_{0}(\alpha)$ that we can obtain the conditional process in $0 \leqq t \leqq T_{0}(\alpha)$ by constructing the one in $0 \leqq t \leqq T_{0}(\beta)$ and then restricting it to $0 \leqq t \leqq T_{0}(\alpha)$. Thus as $\beta \rightarrow \infty$ we obtain a well-defined conditional process in $0 \leqq t<\infty$ whose restriction to $0 \leqq t$ $\leqq T_{0}(\alpha)$, for each $\alpha>0$, is equivalent to the former. Let this process be denoted by $X^{*}(t)$.

If $X^{*}(t)=x$ is now given, $x>0$, we can construct a process equivalent to $X^{*}(t+s), 0 \leqq s$, as follows. Let $Y(t)$ be an unconditional Brownian motion starting at $x>0$ and let $T(0, a)$ denote its first passage time to the set $\{+a, 0\}$. We now condition $Y(t)$ in $0 \leqq t \leqq T(0, a)$ by $Y(T(0, a))=0$, and set $X^{*}(t+s)=Y(s), 0 \leqq s$ $\leqq T(0, a)$. Finally, to define $X^{*}(t+T(0, a)+s), 0 \leqq s \leqq \infty$, we simply use a replica of the whole process $X^{*}(t)$, independent of the past. That this procedure is valid becomes clear upon observing that, conditional upon $t<T_{0}(\beta)$ and $f(t, 0, w)=\alpha$, the process $X^{*}(t+s), 0 \leqq s \leqq T_{0}(\beta)-t$, is obtained from $X(t+s)$ and the condition $\max _{0 \leqq s \leqq T_{0}(\beta)-t}|X(t+s)|<\alpha$ by dividing it into two conditions on disjoint time intervals, one up to the first return time to 0 (which must precede $T_{0}(\beta)-t$ ) and the other from this instant up to $T_{0}(\beta)-t$ during which $f(t+s, 0, w)$ must increase by exactly $\beta-\alpha$, and which by the strong Markov property is therefore independent of the past up to the first return time to 0 .

The conditional process $X^{*}(t+s)$, given $X^{*}(t)=x>0$, is thus equivalent up to its first return to 0 , to $Y(s)$ conditioned by $Y(T(0, a))=0$. Granting that $X^{*}(t)$ is a diffusion process (which is somewhat tedious to verify) this suffices to determine the form of its generator. Since, for $0<x<a$,

$$
P(Y(t) \text { reaches } x+\Delta \text { before } x-\Delta \mid Y(T(0, a))=0)=\frac{1}{2}-\Delta / 2(a-x),
$$

we have as "drift coefficient" the expression $-1 /(a-x)$ for $0<x$, and by symmetry $1 /(a+x)$ for $0>x$. The "speed measure" of a diffusion is determined by properties of the path holding with probability 1 (the "quadratic variation" in the present case) and is not changed through conditioning by a set of positive probability. Thus we can conclude with

( ${ }^{1}$ Added in Proof. This appearance of the Bessel process was discussed in [11, p. 231]. 
THEOREM 3.2. $X^{*}(t)$ is the diffusion in $(-a, a)$ with generator

$$
\frac{1}{2} \frac{d^{2}}{d y^{2}}-\frac{1}{a-y} \frac{d}{d y}
$$

in $(0, a)$, and

$$
\frac{1}{2} \frac{d^{2}}{d y^{2}}+\frac{1}{a+y} \frac{d}{d y}
$$

in $(-a, 0)$.

REMARK. We do not have to specify the generator at $y=0$ since this is a consequence of continuity considerations in the domain of functions whose 2nd derivatives have the required discontinuity at 0 . The same proof shows that the condition $\max _{0 \leqq t \leqq T_{0}(\alpha)} X(t)<a, \alpha \rightarrow \infty$, leads to the process with generator

$$
\frac{1}{2} \frac{d^{2}}{d y^{2}}-\frac{1}{a-y} \frac{d}{d y}
$$

in $(0, a)$, but simply $\frac{1}{2}\left(d^{2} / d y^{2}\right)$ in $(-\infty, 0)$.

\section{REFERENCES}

1. K. L. Chung, Markov chains with stationary transition probabilities, Springer, Berlin, 1960.

2. W. Feller, An introduction to probability theory and its applications, Vol. 1, 2nd ed. and Vol. II, Wiley, New York, 1957 and 1966.

3. K. Ito and H. P. McKean, Jr., Diffusion processes and their sample paths, Springer, Berlin, 1965.

4. F. B. Knight, Random walks and a sojourn density process of Brownian motion, Trans. Amer. Math. Soc. 109 (1963), 56-86.

5. P. Lévy, Processus stochastiques et mouvement brownien, Gauthier-Villars, Paris, 1948.

6. D. B. Ray, Sojourn times of diffusion processes, Illinois J. Math. 7 (1963), 615-630.

7. G. Roberts and H. Kaufman, Tables of Laplace transforms, Saunders, Philadelphia, Pa., 1966.

8. C. Stone, Limit theorems for random walks, birth and death processes, and diffusion processes, Illinois J. Math. 7 (1963), 638-660.

9. H. Trotter, A property of Brownian motion paths, Illinois J. Math. 2 (1958), 425-433.

10. M. Silverstein, A new approach to local times, J. Math. Mech. 17 (1968), 1023-1054.

11. H. P. McKean, Jr., Excursions of a non-singular diffusion, Z. Wahrscheinlichkeitstheorie und Verw. Gebiete 1 (1963), 230-239.

UNIVERSITY OF ILLINOIS, URBANA, ILLINOIS 